\title{
Catalogue of the Star graph eigenvalue multiplicities
}

Received: 2 October 2019 / Accepted: 28 October 2019 / Published online: 19 November 2019 (C) The Author(s) 2019

\begin{abstract}
The Star graph $S_{n}, n \geqslant 2$, is the Cayley graph over the symmetric group $\mathrm{Sym}_{n}$ generated by transpositions $(1 i), 2 \leqslant i \leqslant n$. This set of transpositions plays an important role in the representation theory of the symmetric group. The spectrum of $S_{n}$ contains all integers from $-(n-1)$ to $n-1$, and also zero for $n \geqslant 4$. In this paper we observe methods for getting explicit formulas of eigenvalue multiplicities in the Star graphs $S_{n}$, present such formulas for the eigenvalues $\pm(n-k)$, where $2 \leqslant k \leqslant 12$, and finally collect computational results of all eigenvalue multiplicities for $n \leqslant 50$ in the catalogue.
\end{abstract}

Mathematics Subject Classification $\quad 05 \mathrm{C} 25 \cdot 05 \mathrm{E} 10 \cdot 05 \mathrm{E} 15 \cdot 90 \mathrm{~B} 10$

\section{Introduction}

The Star graph $S_{n}, n \geqslant 2$, is the Cayley graph over the symmetric group $\operatorname{Sym}_{n}$ of permutations $\pi=$ $\left[\pi_{1} \pi_{2} \ldots \pi_{n}\right]$ with the generating set $\left\{(1 i) \in \operatorname{Sym}_{n}: 2 \leqslant i \leqslant n\right\}$ of all transpositions $(1 i)$ swapping the 1 st and the $i$ th elements of a permutation $\pi$. It is a connected bipartite $(n-1)$-regular graph of order $n$ ! and diameter $\operatorname{diam}\left(S_{n}\right)=\left\lfloor\frac{3(n-1)}{2}\right\rfloor[3]$.

A graph is integral if all eigenvalues of its adjacency matrix are integers. In 1974, Harary and Schwenk [10] posed a question on graphs having integral spectra. In general, most of the graphs have nonintegral eigenvalues [2].

In 2000, Friedman [8] investigated the second smallest non-negative eigenvalue $\lambda_{2}$ of Cayley graphs on the symmetric group generated by transpositions. He proved that among all sets of $n-1$ transpositions which generate the symmetric group, the set whose associated Cayley graph has $\lambda_{2}=1$ is the set $\{(12),(13), \ldots,(1 n)\}$. This means that there are no other integral Cayley graphs over the symmetric group generated by sets of $n-1$ transpositions.

In 2009, Abdollahi and Vatandoost conjectured [1] that the spectrum of $S_{n}$ is integral, and contains all integers in the range from $-(n-1)$ up to $n-1$ (with the sole exception that when $n \leqslant 3$, zero is not an

Electronic supplementary material The online version of this article (https://doi.org/10.1007/s40065-019-00271-z) contains supplementary material, which is available to authorized users.

E. Khomyakova $\cdot$ E. V. Konstantinova

Novosibirsk State University, Pirogova str. 2, Novosibirsk 630090, Russia

E-mail: ekhomnsu@gmail.com

E. V. Konstantinova ( $\square)$

Sobolev Institute of Mathematics, Ak. Koptyug av. 4, Novosibirsk 630090, Russia

E-mail: e_konsta@math.nsc.ru 
eigenvalue of $S_{n}$ ). Partially this conjecture was based on the known fact about the spectrum of an $r$-regular graph which lies in the segment $[-r, r]$ [5]. They verified this conjecture numerically using GAP for $n \leqslant 6$.

In 2012, Krakovski and Mohar [14] proved the second part of the conjecture. More precisely, they proved that for $n \geqslant 2$ and for each integer $1 \leqslant k \leqslant n-1$, the values $\pm(n-k)$ are eigenvalues of $S_{n}$ with multiplicity at least $\left(\begin{array}{l}n-2 \\ k-1\end{array}\right)$. If $n \geqslant 4$, then 0 is an eigenvalue of $S_{n}$ with multiplicity at least $\left(\begin{array}{c}n-1 \\ 2\end{array}\right)$. Since the Star graph is bipartite, $\operatorname{mul}(n-k)=\operatorname{mul}(-n+k)$ for each integer $1 \leqslant k \leqslant n$. Moreover, $\pm(n-1)$ are simple eigenvalues of $S_{n}$.

At the same time, Chapuy and Feray [6] showed that the integrality of the Star graphs was already solved in another context, since it is equivalent to studying the spectrum of Jucys-Murphy elements in the algebra of the symmetric group [11]. This connection between two kinds of spectra implies that the Star graph is integral. References on the topic can be also found in the introduction of the paper by Renteln [15].

A lower bound on multiplicities of eigenvalues of $S_{n}$ given by Krakovski and Mohar was improved by Chapuy and Feray as follows:

$$
\operatorname{mul}(n-k) \geqslant\left(\begin{array}{c}
n-2 \\
n-k-1
\end{array}\right)\left(\begin{array}{l}
n-1 \\
n-k
\end{array}\right) .
$$

In 2016, Avgustinovich et al. [4,12] suggested a method for getting explicit formulas for multiplicities of eigenvalues $\pm(n-k)$ in the Star graphs $S_{n}$ and presented such formulas for $2 \leqslant k \leqslant 5$. Moreover, a lower bound on multiplicity of eigenvalues of $S_{n}$ for sufficiently large $n$ was obtained. It was proved that for a fixed integer eigenvalue of the Star graph $S_{n}$, its multiplicity is at least $2^{\frac{1}{2} n \log n(1-o(1))}$ [4].

In 2018, Khomyakova [13] investigated the behavior of the eigenvalues multiplicity function of the Star graph $S_{n}$ for eigenvalues $\pm(n-k)$ where $1 \leqslant k \leqslant \frac{n+1}{2}$. It was shown that the function has a polynomial behavior in $n$. Moreover, explicit formulas for calculating multiplicities of eigenvalues $\pm(n-k)$ where $2 \leqslant k \leqslant 12$ were also presented in the paper. Computational results showed that the same polynomial behavior of the eigenvalues multiplicity function occurs for any integers $n \geqslant 2$ and $1 \leqslant k \leqslant n$.

In this paper, we review methods used for getting explicit formulas for eigenvalue multiplicities in the Star graphs $S_{n}$, present these formulas for the eigenvalues $\pm(n-k)$, where $2 \leqslant k \leqslant 12$, and finally collect computational results of all eigenvalue multiplicities for $n \leqslant 50$ in the catalogue provided in the electronic supplementary material.

\section{Theoretical results}

To describe a combinatorial approach for calculating multiplicities of eigenvalues of the Star graphs $S_{n}, n \geqslant 2$, we need to give basic definitions and notation on representation theory of the symmetric group [16].

The symmetric group $\mathrm{Sym}_{n}$ consists of all bijections of $\{1,2, \ldots, n\}$ to itself using compositions as the multiplication. For any permutation $\pi \in S y m_{n}$, we view its cycle type as a partition.

A partition of $n$ is a sequence $\lambda=\left(\lambda_{1}, \lambda_{2}, \ldots, \lambda_{l}\right), l \leqslant n$, such that $\lambda_{1} \geqslant \lambda_{2} \geqslant \cdots \geqslant \lambda_{l}$ and $\lambda_{1}+\lambda_{2}+\cdots+\lambda_{l}=n$. We denote a partition of $n$ as $\lambda \vdash n$. If $\pi \in \operatorname{Sym}_{n}$ is decomposed into a product of disjoint cycles of length $\lambda_{1}, \ldots, \lambda_{l}$, where $\lambda_{1} \geqslant \cdots \geqslant \lambda_{l}$ so that $\lambda_{1}+\cdots+\lambda_{l}=n$, then a partition $\lambda=\left(\lambda_{1}, \lambda_{2}, \ldots, \lambda_{l}\right)$ is called the cycle type of $\pi$. A partition $\lambda$ is presented by its Young diagram. In this paper, we use the French notation for Young diagrams [9].

Let $\lambda$ is a partition of $n$. A Young tableau of shape $\lambda$ is obtained by filling in the boxes of a Young diagram of $\lambda$ with the elements $\{1,2, \ldots, n\}$, where each number occurring exactly once. Thus, the Young tableau of shape $\lambda$ is the set $[\lambda]=\left\{(i, j): 1 \leqslant j \leqslant \lambda_{i}, 1 \leqslant i \leqslant l\right\}$. Let us define values $c(m)=i-j$, where $m \in\{1, \ldots, n\}$ and $i, j$ are the ordinate and the abscissa of the box containing $m$, correspondingly. A standard Young tableau is a Young tableau whose the entries are increasing across each row and each column.

We write $\lambda^{\prime}$ for the conjugate partition of $\lambda$ defined by $\lambda^{\prime}=\left(\lambda_{1}^{\prime}, \lambda_{2}^{\prime}, \ldots, \lambda_{l^{\prime}}^{\prime}\right)$, where $l^{\prime}=\lambda_{1}, \lambda_{j}^{\prime}=$ $\max \{j:(i, j) \in[\lambda]\}, 1 \leqslant i \leqslant l^{\prime}$. So, $(i, j) \in[\lambda]$ if and only if $(j, i) \in\left[\lambda^{\prime}\right]$. Then, the hook length $h_{i j}$ is defined by the following formula:

$$
h_{i j}=\lambda_{i}-j+\lambda_{j}^{\prime}-i+1 .
$$

Now let us show relationships between standard Young tableaux and eigenvalue multiplicities of the Star graphs. 
Let $G$ be a group and $V$ be a finite-dimensional vector space over the complex numbers. Let $G L(V)$ stands for the set of all invertible linear transformations of $V$ to itself, called the general linear group of $V$. Then a representation of $G$ on $V$ is a group homomorphism $\rho: G \rightarrow G L(V)$, and $V$ is a vector space of the representation with dimension $\operatorname{dim}(V)$. The representation is irreducible if it has no proper subspace closed under the action of $\rho$. Two representations $\rho_{1}: G \rightarrow G L\left(V_{1}\right)$ and $\rho_{2}: G \rightarrow G L\left(V_{2}\right)$ are equivalent if there exists a bijective linear map $\varphi: V_{1} \rightarrow V_{2}$ such that $\varphi \rho_{2}(g)=\rho_{1}(g) \varphi$ for all $g \in G$.

The symmetric group $\operatorname{Sym}_{n}$ has order $n$ !, its conjugacy classes are labeled by partitions of $n$, and according to the representation theory of a finite group, the set of inequivalent irreducible representations is defined by partitions of $n$. We denote by $V_{\lambda}$ a vector space of the irreducible representation associated with the partition $\lambda \vdash n$. It is known [16] that

$$
\sum_{\lambda \vdash n}\left(\operatorname{dim}\left(V_{\lambda}\right)\right)^{2}=\left|\operatorname{Sym}_{n}\right|
$$

and the following equality holds [6]

$$
\operatorname{mul}(n-k)=\sum_{\lambda \vdash n} \operatorname{dim}\left(V_{\lambda}\right) I_{\lambda}(n-k)
$$

where $I_{\lambda}(n-k)$ is the number of standard Young tableaux of shape $\lambda$ satisfying $c(n)=n-k$. Since $\operatorname{dim}\left(V_{\lambda}\right)$ is equal to the number of all partitions of shape $\lambda$, i.e., the number of standard Young tableaux, it is calculated by the Hook Formula [7]:

$$
\operatorname{dim}\left(V_{\lambda}\right)=\frac{n !}{\prod_{(i, j) \in[\lambda]} h_{i j}},
$$

where $\lambda \vdash n$. Let $\mathcal{A}_{k}$ be the set of partitions of $n$ of length $l=n-k+1$ with the last element 1 . For any $\lambda=\left(\lambda_{1}, \ldots, \lambda_{n-k}, 1\right)$, let $\hat{\lambda}$ be a partition $\left(\lambda_{1}, \ldots, \lambda_{n-k}\right)$ of $n-1$. Then the following result holds.

Lemma 2.1 [13] For any integer $k, 1 \leqslant k \leqslant \frac{n+1}{2}$, we have

$$
I_{\lambda}(n-k)= \begin{cases}\operatorname{dim}\left(V_{\hat{\lambda}}\right), & \text { if } \lambda \in \mathcal{A}_{k} \\ 0, & \text { if } \lambda \notin \mathcal{A}_{k} .\end{cases}
$$

We set $\lambda=\left(\lambda_{1}, \lambda_{2}, \ldots, \lambda_{l}\right) \vdash n, l=n-k+1, \lambda \in \mathcal{A}_{k}$, and calculate the hook length $\widehat{h_{i j}}$ in $[\hat{\lambda}]$ by the following formulas:

$$
\widehat{h_{i j}}= \begin{cases}\lambda_{i}-j+\left(\lambda_{j}^{\prime}-1\right)-i+1, & j=1,1 \leqslant i \leqslant l-1 \\ \lambda_{i}-j+\lambda_{j}^{\prime}-i+1, & 1<j \leqslant \lambda_{i}, 1 \leqslant i \leqslant l-1 ; \\ 0, & i=l=n-k+1 .\end{cases}
$$

Then by Lemma 2.1, for any $k, 1 \leqslant k \leqslant \frac{n+1}{2}$, formula (4) can be rewritten as:

$$
\operatorname{mul}(n-k)=\sum_{\lambda \in \mathcal{A}_{k}} \frac{n !}{\prod_{(i, j) \in[\lambda]} h_{i j}} \cdot \frac{(n-1) !}{\prod_{(i, j) \in[\hat{\lambda}]} \widehat{h_{i j}}} .
$$

The main result is given by the following theorem.

Theorem 2.2 [13] Let $n, k \in \mathbb{Z}, n \geqslant 2$ and $1 \leqslant k \leqslant \frac{n+1}{2}$, then the multiplicity $\operatorname{mul}(n-k)$ of eigenvalue $(n-k)$ of the Star graph $S_{n}$ is calculated by the formula:

$$
\operatorname{mul}(n-k)=\frac{n^{2(k-1)}}{(k-1) !}+P(n)
$$

where $P(n)$ is a polynomial of degree $2 k-3$.

Computational results show that theorem holds for any $n \geqslant 2$ and $1 \leqslant k \leqslant n$. 


\section{Practical results}

Explicit formulas of multiplicities for the positive eigenvalues $(n-k)$, where $2 \leqslant k \leqslant 12$, are obtained by (8) and given in "Appendix A". Since the Star graph $S_{n}$ is bipartite, the same formulas hold for negative eigenvalues $-(n-k)$. The results of our computations are summarized in electronic supplementary material. To get these results an algorithm based on the method above was implemented in Golang. The calculations were performed on the Intel(R) Core(TM) i7-4790K CPU @ 4.00GHz with the following elapsed time:

\begin{tabular}{|c|c|c|c|c|c|c|c|c|c|c|c|}
\hline$n$ & 50 & 55 & 60 & 65 & 70 & 75 & 80 & 85 & 90 & 95 & 100 \\
\hline $\mathrm{sec}$ & 1.5 & 3.6 & 8.2 & 18.6 & 40.6 & 87 & 181.8 & 373.1 & 745.5 & 1461.9 & 2827.9 \\
\hline
\end{tabular}

Acknowledgements The authors thank the anonymous referee whose comments and suggestions allowed to improve the manuscript. The reported study was funded by RFBR according to the research projects 17-51-560008 and 18-01-00353. The work was supported by the program of fundamental scientific research of the SB RAS N I.5.1, project No.0314-2019-0016.

Open Access This article is distributed under the terms of the Creative Commons Attribution 4.0 International License (http:// creativecommons.org/licenses/by/4.0/), which permits unrestricted use, distribution, and reproduction in any medium, provided you give appropriate credit to the original author(s) and the source, provide a link to the Creative Commons license, and indicate if changes were made.

\section{Appendix A: Explicit formulas of multiplicities $(n-k)$ for $2 \leqslant k \leqslant 12$}

$$
\begin{aligned}
& \operatorname{mul}(n-2)=(n-1)(n-2) . \\
& \operatorname{mul}(n-3)=\frac{(n-1)(n-3)}{2 !}\left(n^{2}-4 n+2\right) \text {. } \\
& \operatorname{mul}(n-4)=\frac{(n-1)(n-2)}{3 !}\left(n^{4}-12 n^{3}+47 n^{2}-62 n+12\right) \text {. } \\
& \operatorname{mul}(n-5)=\frac{(n-1)(n-2)}{4 !}\left(n^{6}-21 n^{5}+169 n^{4}-647 n^{3}+1174 n^{2}-820 n+60\right) . \\
& \operatorname{mul}(n-6)=\frac{(n-1)(n-2)(n-3)}{5 !}\left(n^{7}-29 n^{6}+335 n^{5}-1955 n^{4}+6004 n^{3}-9028 n^{2}+5068 n-120\right) . \\
& \operatorname{mul}(n-7)=\frac{(n-1)(n-2)(n-3)}{6 !}\left(n^{9}-42 n^{8}+744 n^{7}-7228 n^{6}+41889 n^{5}\right. \\
& \left.-147190 n^{4}+302970 n^{3}-328600 n^{2}+140336 n-840\right) \text {. } \\
& \operatorname{mul}(n-8)=\frac{(n-1)(n-2)(n-3)(n-4)}{7 !}\left(n^{10}-53 n^{9}+1206 n^{8}-15400 n^{7}+120995 n^{6}-602539 n^{5}\right. \\
& \left.+1884770 n^{4}-3526192 n^{3}+3515644 n^{2}-1385752 n+1680\right) \text {. } \\
& \operatorname{mul}(n-9)=\frac{(n-1)(n-2)(n-3)(n-4)}{8 !}\left(n^{12}-70 n^{11}+2165 n^{10}-38962 n^{9}+452071 n^{8}\right. \\
& -3538962 n^{7}+18992119 n^{6}-69486678 n^{5}+168611532 n^{4}-255823264 n^{3} \\
& \left.+215298016 n^{2}-74535168 n+15120\right) \text {. } \\
& \operatorname{mul}(n-10)=\frac{(n-1)(n-2)(n-3)(n-4)(n-5)}{9 !}\left(n^{13}-84 n^{12}+3149 n^{11}-69516 n^{10}+1003947 n^{9}\right. \\
& -9962604 n^{8}+69399287 n^{7}-340356732 n^{6}+1159537744 n^{5}-2655899000 n^{4} \\
& \left.+3843130464 n^{3}-3110922016 n^{2}+1044298320 n-30240\right) \text {. } \\
& \operatorname{mul}(n-11)=\frac{(n-1)(n-2)(n-3)(n-4)(n-5)}{10 !}\left(n^{15}-105 n^{14}+5005 n^{13}-143355 n^{12}+2752087 n^{11}\right. \\
& -37392489 n^{10}+370002755 n^{9}-2701681905 n^{8}+14581677068 n^{7}-57662568122 n^{6}
\end{aligned}
$$




$$
\begin{aligned}
& +163637288684 n^{5}-321068683400 n^{4}+408207829104 n^{3} \\
& \left.-297483438704 n^{2}+92156167776 n-332640\right) . \\
\operatorname{mul}(n-12)= & \frac{(n-1)(n-2)(n-3)(n-4)(n-5)(n-6)}{11 !}\left(n^{16}-122 n^{15}+6800 n^{14}-229410 n^{13}\right. \\
& +5231382 n^{12}-85270438 n^{11}+1024324268 n^{10}-9213080470 n^{9}+62383290673 n^{8} \\
& -316945929608 n^{7}+1193507219068 n^{6}-3254674992768 n^{5}+6181444807824 n^{4} \\
& \left.-7653794621472 n^{3}+5460913410224 n^{2}-1664568429792 n+665280\right) .
\end{aligned}
$$

\section{References}

1. Abdollahi, A.; Vatandoost, E.: Which Cayley graphs are integral? Electron. J. Combin. 16, 6-7 (2009)

2. Ahmadi, O.; Alon, N.; Blake, I.F.; Shparlinski, I.E.: Graphs with integral spectrum. Linear Algebra Appl. 430, 547-552 (2009)

3. Akers, S.B.; Krishnamurthy, B.: A group-theoretic model for symmetric interconnection networks. IEEE Trans. Comput. 38(4), 555-566 (1989)

4. Avgustinovich, S.V.; Khomyakova, E.N.; Konstantinova, E.V.: Multiplicities of eigenvalues of the Star graph. Siber. Electron. Math. Rep. 13, 1258-1270 (2016)

5. Brouwer, A.E.; Haemers, W.H.: Spectra of Graphs. Springer, New York (2012)

6. Chapuy, G.; Feray, V.: A note on a Cayley graph of Sym . arXiv:1202.4976v2 1-3 (2012)

7. Frame, J .S.; Robinson, G de B; Thrall, R.M.: The hook graphs of the symmetric group. Can. J. Math. 6, 316-325 (1954)

8. Friedman, J.: On Cayley graphs on the symmetric group generated by transpositions. Combinatorica 20(4), 505-519 (2000)

9. Fulton, W.: Young Tableaux: With Applications to Representation Theory and Geometry, London Mathematical Society Student Texts. Cambridge University Press, Cambridge (1996). https://doi.org/10.1017/CBO9780511626241

10. Harary, F.; Schwenk, A.J.: Which graphs have integral spectra? Graphs Combin. 390, 45-51 (1974)

11. Jucys, A.: Symmetric polynomials and the center of the symmetric group ring. Reports Math. Phys. 5, 107-112 (1974)

12. Khomyakova, E.N.; Konstantinova, E.V.: Note on exact values of multiplicities of eigenvalues of the Star graph. Siber. Electron. Math. Rep. 12, 92-100 (2015)

13. Khomyakova, E.N.: On the eigenvalues multiplicity function of the Star graph. Siber. Electron. Math. Rep. 15, 1416-1425 (2018)

14. Krakovski, R.; Mohar, B.: Spectrum of Cayley graphs on the symmetric group generated by transposition. Linear Algebra Appl. 437, 1033-1039 (2012)

15. Renteln, P.: The distance spectra of Cayley graphs of Coxeter groups. Discrete Math. 311, 738-755 (2011)

16. Sagan, B.: The Symmetric Group: Representations, Combinatorial Algorithms, and Symmetric Functions, 2nd edn. Springer, New York (2001)

Publisher's Note Springer Nature remains neutral with regard to jurisdictional claims in published maps and institutional affiliations. 